\title{
Heart disease prediction using machine learning techniques : a survey
}

\author{
V.V. Ramalingam*, Ayantan Dandapath, M Karthik Raja \\ ${ }^{1,2}$ Department of Computer Science \& Engineering, SRM Institute of Science and Technology \\ *Corresponding Author E-mail: ramalingam.v@ktr.srmuniv.ac.in
}

\begin{abstract}
Heart related diseases or Cardiovascular Diseases (CVDs) are the main reason for a huge number of death in the world over the last few decades and has emerged as the most life-threatening disease, not only in India but in the whole world. So, there is a need of reliable, accurate and feasible system to diagnose such diseases in time for proper treatment. Machine Learning algorithms and techniques have been applied to various medical datasets to automate the analysis of large and complex data. Many researchers, in recent times, have been using several machine learning techniques to help the health care industry and the professionals in the diagnosis of heart related diseases. This paper presents a survey of various models based on such algorithms and techniques andanalyze their performance. Models based on supervised learning algorithms such as Support Vector Machines (SVM), K-Nearest Neighbour (KNN), NaïveBayes, Decision Trees (DT), Random Forest (RF) and ensemble models are found very popular among the researchers.
\end{abstract}

Keywords: Cardiovascular Diseases; Support Vector Machines; K- Nearest Neighbour; Nä̈ve Bayes; Decision Tree; Random Forest; Ensemble Models.

\section{Introduction}

Heart is an important organ of the human body. It pumps blood to every part of our anatomy. If it fails to function correctly, then the brain and various other organs will stop working, and within few minutes, the person will die. Change in lifestyle, work related stress and bad food habits contribute to the increase in rate of several heart related diseases.

Heart diseases have emerged as one of the most prominent cause of death all around the world. According to World Health Organisation, heart related diseases are responsible for the taking 17.7 million lives every year, $31 \%$ of all global deaths. In India too, heart related diseases have become the leading cause of mortality [1]. Heart diseases have killed 1.7 million Indians in 2016, according to the 2016 Global Burden of Disease Report, released on September 15,2017. Heart related diseases increase the spending on health care and also reduce the productivity of an individual. Estimates made by the World Health Organisation (WHO), suggest that India have lost up to \$237 billion, from 2005-2015, due to heart related or Cardiovascular diseases [2]. Thus, feasible and accurate prediction of heart related diseases is very important.

Medical organisations, all around the world, collect data on various health related issues. These data can be exploited using various machine learning techniques to gain useful insights. But the data collected is very massive and, many a times, this data can be very noisy. These datasets, which are too overwhelming for human minds to comprehend, can be easily explored using various machine learning techniques. Thus, these algorithms have become very useful, in recent times, to predict the presence or absence of heart related diseases accurately.

\section{Dimensionality Reduction}

Dimensionality Reduction involves selecting amathematical representation such that one can relate the majority of, but not all, the variance within the given data, thereby including only most significant information. The data considered for a task or a problem, may consists of a lot of attributesor dimensions, but not all of these attributes may equally influence the output. A large number of attributes, or features, may affect the computational complexity and may even lead to overfitting which leads to poor results. Thus, Dimensionality Reduction is a very important step considered while building any model. Dimensionality Reduction is generally achieved by two methods -Feature Extraction and Feature Selection.

\section{A. Feature Extraction}

In this, a new set of features is derived from the original feature set.Feature extraction involves a transformation of the features. This transformation is often not reversible asfew, or maybe many, useful information is lost in the process.In [3]and[4]Principal Component Analysis (PCA)is used for feature extraction. Principal Component Analysis is a popularly used linear transformation algorithm. In the feature space, it finds the directions that maximize variance and finds directions that are mutually orthogonal. It is a global algorithm that gives the best reconstruction.

\section{B. Feature Selection}

In this, a subset of original feature set is selected. In [5],key features are selected by CFS(Correlation based Feature Selection) Subset Evaluation combined with Best First Search method to reduce dimensionality. In [6]chi-square statistics test is used to select the most significant features. 


\section{Algorithms and Techniques Used}

\section{A. Naïve Bayes}

Naive Bayes is a simple but an effective classification technique which is based on the Bayes Theorem. It assumes independence among predictors, i.e., the attributes or features should be not correlated to one another or should not, in anyway, be related to each other. Even if there is dependency, still all these features or attributes independently contribute to the probability and that is whv it is called Naïve

$$
\begin{gathered}
P(c \mid x)=\frac{P(x \mid c) P(c)}{P(x)} \\
P(c \mid \mathrm{X})=P\left(x_{1} \mid c\right) \times P\left(x_{2} \mid c\right) \times \cdots \times P\left(x_{n} \mid c\right) \times P(c)
\end{gathered}
$$

In [7], Naive Bayes has achieved an accuracy of $84.1584 \%$ with the 10 most significant features which are selected using SVMRFE (Recursive Feature Elimination) and gain ratio algorithms whereas in[8],Naive Bayes has achieved an accuracy of $83.49 \%$ when all 13 attributes of the Cleveland dataset[25] are used.

\section{B. Support Vector Machine}

Support Vector Machine is an extremely popular supervised machine learning technique(having a pre-defined target variable) which can be used as a classifier as well as a predictor. For classification, it finds a hyper-plane in the feature space that differentiates between the classes. An SVM model represents the training data points as points in the feature space, mapped in such a way that points belonging to separate classes are segregated by a margin as wide as possible. The test data points are then mapped into that same space and are classified based on which side of the margin they fall.

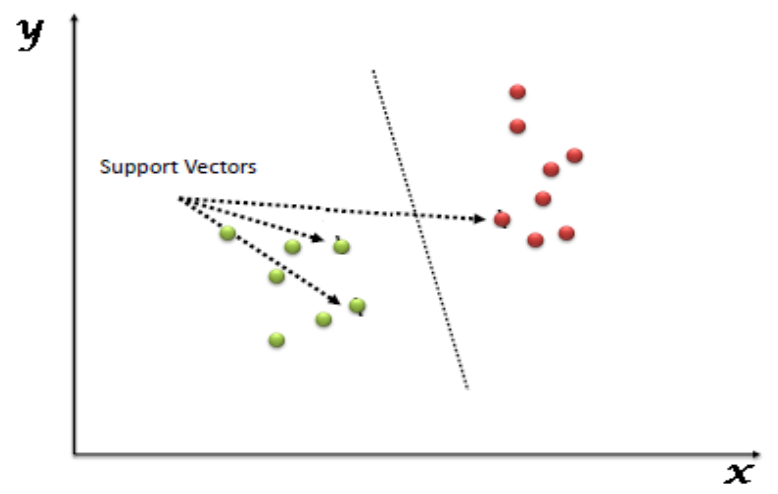

Fig. 1: Support Vector Machine

Shan Xu et al. have used SVM to achieve an accuracy of $98.9 \%$ in People's Hospital dataset [5].In [9], SVM performs the best with $85.7655 \%$ of correctly classified instance and in [10] SVM is used with boosting technique to give an accuracy of $84.81 \%$. HoudaMezrigui et al. have used SVM to attain a f-measure value of 93.5617 [11]. In [12] SVM classifies the pixel variation with an accuracy of $92.1 \%$ helping to identify the affected region accurately.

\section{C. $\mathbf{K}$ - Nearest Neighbour}

In 1951, Hodges et al. introduced a nonparametric technique for pattern classification which is popularly known the K-Nearest Neighbour rule[13]. K-Nearest Neighbour technique is one of the most elementary but very effective classification techniques. It makes no assumptions about the data and is generally be used for classification tasks when there is very less or no prior knowledge about the data distribution. This algorithm involves finding the $\mathrm{k}$ nearest data points in the training set to the data point for which a target value is unavailable and assigning the average value of the found data points to it.

In [10] KNN gives an accuracy of $83.16 \%$ when the value of $\mathrm{k}$ is equal to 9 while using 10-cross validation technique. In [14]

KNN with Ant Colony Optimization performs better than other techniques with an accuracy of $70.26 \%$ and the error rates is 0.526.Ridhi Saini et al. have obtained a efficiency of $87.5 \%$ [15], which is very good.

\section{Decision Tree}

Decision tree is a of supervised learning algorithm.This technique is mostly used in classification problems. It performs effortlessly withcontinuous and categorical attributes. This algorithm dividesthe population into two or more similar sets based on the most significantpredictors.Decision Treealgorithm, first calculates the entropy of each and every attribute. Then the dataset is split with the help of thevariables or predictors with maximum information gain or minimum entropy. These two steps are performed recursively with the remaining attributes.

$$
\begin{aligned}
& \operatorname{Entropy}(S)=\sum_{i=1}^{c}-p_{i} \log _{2} p_{i} \\
& \operatorname{Gain}(S, A)=\operatorname{Entropy}(S)-\sum_{v \in \operatorname{Values}(A)} \frac{\left|S_{v}\right|}{|S|} \operatorname{Entropy}\left(S_{v}\right)
\end{aligned}
$$

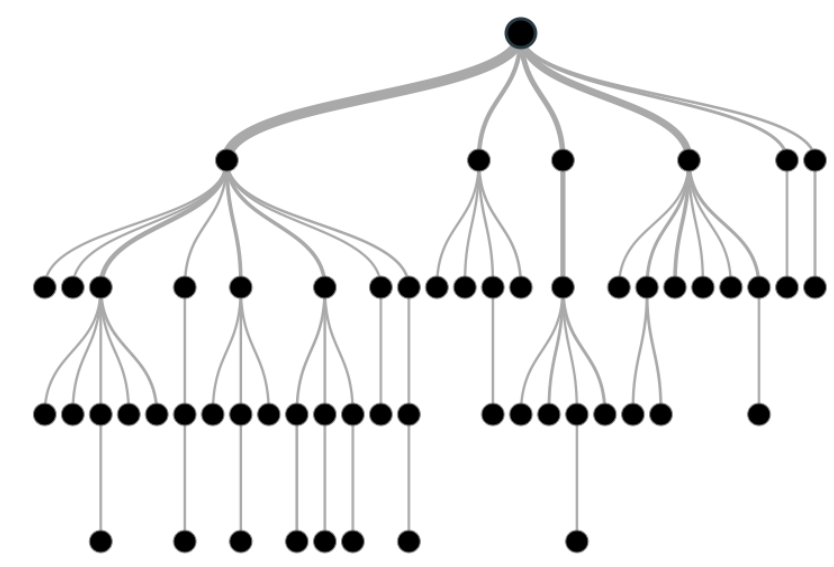

Fig. 2: Decision Tree

In [10]decision tree has the worst performance with an accuracy of $77.55 \%$ but when decision tree is used with boosting technique it performs better with an accuracy of $82.17 \%$.In [9] decision tree performs very poorly with a correctly classified instance percentage of $42.8954 \%$ whereas in [16] also uses the same dataset but used the J48 algorithm for implementing Decision Trees and the accuracy thus obtained is $67.7 \%$ which is less but still an improvement on the former. Renu Chauhan et al. have obtained an accuracy of $71.43 \%$ [17]. M.A. Jabbar et al. have used alternating decision trees with principle component analysis to obtain an accuracy 92.2\%[18].Kamran Farooq et al. have achieved the best results on using decision tree-based classifier combined with forward selection which achieves a weighted accuracy of $78.4604 \%$ [19]. 


\section{E. Random Forest}

Random Forest is also a popularly supervised machine learning algorithm.This technique can be used for both regression and classification tasks but generally performs better in classification tasks. As the name suggests, Random Forest technique considers multiple decision trees before giving an output. So, it is basically an ensemble of decision trees. This technique is based on the belief that more number of trees would converge to the right decision. For classification, it uses a voting system and then decides the class whereas in regression it takes the mean of all the outputs of each of the decision trees. It works well with large datasets with high dimensionality.

\section{Random Forest Simplified}

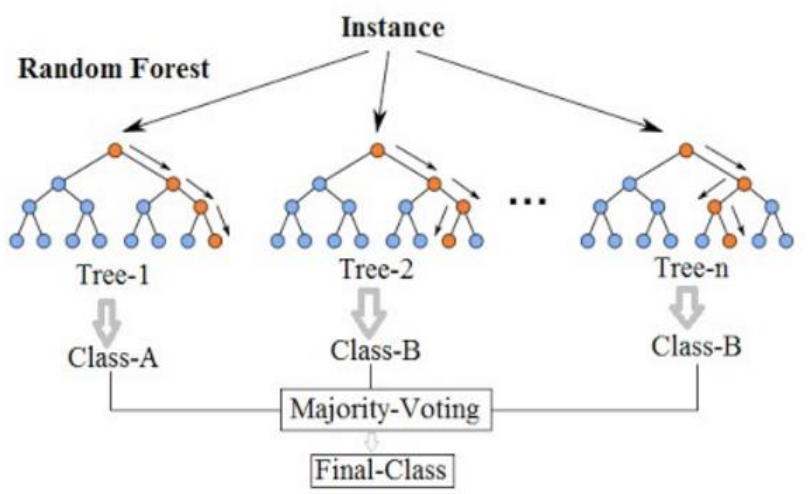

Fig. 3: Random Forest

In [5], random forest performs exceptionally well. In Cleveland dataset, random forest has a significantly higher accuracy of 91.6\% than all the other methods. In People's Hospital dataset, it achieves an accuracy of 97\%. In [20] random forest has achieved an f-measure of 0.86 . In [21], random forest is used to predict coronary heart disease and it obtains an accuracy of $97.7 \%$.

\section{F. Ensemble Model}

In ensemble modeling two or more related but different analytical models are used and produce their results are combined into a single score.

TahiraMahboob et al. [22] have used an ensemble of SVM, KNN and ANN to achieve an accuracy of $94.12 \%$. The Majority votebased model as demonstrated by Saba Bashir et al. [23] which comprises of Naïve Bayes, Decision Tree and Support Vector Machine classifiers, gave an accuracy of $82 \%$, sensitivity of $74 \%$ and specificity of $93 \%$ for UCI heart disease dataset. In [24] an ensemble model, consisting ofGini Index,SVMand Naïve Bayes classifiers, has been proposed which gave an accuracy of $98 \%$ in predicting Syncope disease.

\section{Conclusion}

Based on the above review, it can be concluded that there is a huge scope for machine learning algorithms in predicting cardiovascular diseases or heart related diseases. Each of the above-mentioned algorithms have performed extremely well in some cases but poorly in some other cases. Alternating decision trees when used with PCA, have performed extremely well but decision trees have performed very poorly in some other cases which could be due to overfitting. Random Forest and Ensemble models have performed very well because they solve the problem of overfitting by employing multiple algorithms (multiple Decision Trees in case of Random Forest). Models based on Naïve Bayes classifier were computationally very fast and have also performed well.SVM performed extremely well for most of the cases. Systems based on machine learning algorithms and techniques have been very accurate in predicting the heart related diseases but still there is a lot scope of research to be done on how to handle high dimensional data and overfitting. A lot of research can also be done on the correct ensemble of algorithms to use for a particular type of data.

\section{Acknowledgment}

We sincerely thank the staff of SRM Institute of Science and Technology, that have provided their immense support and guidance throughout the project.

\section{Reference}

[1] Ramadoss and Shah B et al."A. Responding to the threat of chronic diseases in India”. Lancet. 2005; 366:1744-1749. doi 10.1016/S0140-6736(05)67343-6

[2] Global Atlas on Cardiovascular Disease Prevention and Control Geneva, Switzerland: World Health Organization, 2011

[3] Dhomse Kanchan B and Mahale Kishor M. et al. "Study of Machine Learning Algorithms for Special Disease Prediction using Principal of Component Analysis", 2016 International Conference on Global Trends in Signal Processing, Information Computing and Communication.

[4] R.Kavitha and E.Kannan et al. "An Efficient Framework for Heart Disease Classification using Feature Extraction and Feature Selection Technique in Data Mining “, 2016

[5] Shan Xu ,Tiangang Zhu, Zhen Zang, Daoxian Wang, Junfeng Hu and Xiaohui Duan et al. "Cardiovascular Risk Prediction Method Based on CFS Subset Evaluation and Random Forest Classification Framework", 2017 IEEE 2nd International Conference on Big Data Analysis.

[6] Manpreet Singh, Levi Monteiro Martins, Patrick Joanis and Vijay K. Mago et al. " Building a Cardiovascular Disease Predictive Model using Structural Equation Model \& Fuzzy Cognitive Map”, 978-1-5090-0626-7/16/\$31.00 c 2016 IEEE.

[7] Kanika Pahwa and Ravinder Kumar et al. "Prediction of Heart Disease Using Hybrid Technique For Selecting Features", 2017 4th IEEE Uttar Pradesh Section International Conference on Electrical, Computer and Electronics (UPCON).

[8] Seyedamin Pouriyeh, Sara Vahid, Giovanna Sannino, Giuseppe De Pietro, Hamid Arabnia, Juan Gutierrez et al. “ A Comprehensive Investigation and Comparison of Machine Learning Techniques in the Domain of Heart Disease", 22nd IEEE Symposium on Computers and Communication (ISCC 2017): Workshops - ICTS4eHealth 2017

[9] Hanen Bouali and Jalel Akaichi et al. "Comparative study of Different classification techniques, heart Diseases use Case.", 2014 13th International Conference on Machine Learning and Applications

[10] Seyedamin Pouriyeh, Sara Vahid, Giovanna Sannino, Giuseppe De Pietro, Hamid Arabnia, Juan Gutierrez et al. " A Comprehensive Investigation and Comparison of Machine Learning Techniques in the Domain of Heart Disease", 22nd IEEE Symposium on Computers and Communication (ISCC 2017): Workshops - ICTS4eHealth 2017

[11] Houda Mezrigui, Foued Theljani and Kaouther Laabidi et al. "Decision Support System for Medical Diagnosis Using a KernelBased Approach", ICCAD'17, Hammamet - Tunisia, January 1921, 2017.

[12] Dr.(Mrs).D.Pugazhenthi, Quaid-E-Millath and Meenakshi et al. "Detection Of Ischemic Heart Diseases From Medical Images " 2016 International Conference on Micro-Electronics and Telecommunication Engineering.

[13] J. Hodges et al. "Discriminatory analysis, nonparametric discrimination: Consistency properties," 1981.

[14] S.Rajathi and Dr.G.Radhamani et al. "Prediction and Analysis of Rheumatic Heart Disease using kNN Classification with ACO “, 2016.

[15] Puneet Bansal and Ridhi Saini et al. "Classification of heart diseases from ECG signals using wavelet transform and kNN classifier", International Conference on Computing, Communication and Automation (ICCCA2015).

[16] Simge EKIZ and Pakize Erdogmus et al. "Comparitive Study of heart Disease Classification", 978-1-5386-0440-3/17/\$31.00 (C)2017 IEEE.

[17] Renu Chauhan, Pinki Bajaj, Kavita Choudhary and Yogita Gigras et al. "Framework to Predict Health Diseases Using Attribute 
Selection Mechanism", 2015 2nd International Conference on Computing for Sustainable Global Development (INDIA Com).

[18] M.A.JABBAR , B.L Deekshatulu and Priti Chndra et al "Alternating decision trees for early diagnosis of heart disease", Proceedings of International Conference on Circuits, Communication, Control and Computing (I4C 2014).

[19] Amir Hussain, Peipei Yang, Mufti Mahmud and Jan Karasek et al. "A Novel Cardiovascular Decision Support Framework for effective clinical Risk Assessment.”, 978-1-4799-45276/14/\$31.00 @2014 IEEE.

[20] Quazi Abidur Rahman, Larisa G. Tereshchenko, Matthew Kongkatong, Theodore Abraham, M. Roselle Abraham, and Hagit Shatkay et al. "Utilizing ECG-based Heartbeat Classification for Hypertrophic Cardiomyopathy Identification", DOI 10.1109/TNB.2015.2426213, IEEE Transactions on Nano Bioscience TNB-00035-2015.

[21] Ahmad Shahin, Walid Moudani, Fadi Chakik, Mohamad Khalil et al. "Data Mining in Healthcare Information Systems: Case Studies in Northern Lebanon", ISBN: 978-1-4799-3166-8 C2014 IEEE.

[22] Tahira Mahboob, Rida Irfan and Bazelah Ghaffar et al. "Evaluating Ensemble Prediction of Coronary Heart Disease using Receiver Operating Characteristics", 978-1-5090-48151/17/\$31.00 @2017 IEEE.

[23] Saba Bashir, Usman Qamar, M.Younus Javed et al. “An Ensemble based Decision Support Framework for Intelligent Heart Disease Diagnosis" International Conference on Information Society (iSociety 2014)

[24] Ammar Asjad Raja, Irfan-ul-Haq, Madiha Guftar Tamim Ahmed Khan and Dominik Greibl et al. "Intelligent Syncope Disease Prediction Framework using DM-Ensemble Techniques", FTC 2016 - Future Technologies Conference 2016.

[25] CI Education, Heart Disease Data Set [OL]. http://archive.ics.uci.edu/ml/datasets/Heart+Disease CHDD.

[26] T. Padmapriya and V.Saminadan, "Handoff Decision for Multiuser Multiclass Traffic in MIMO-LTE-A Networks", 2nd International Conference on Intelligent Computing, Communication \& Convergence (ICCC-2016) - Elsevier PROCEDIA OF COMPUTER SCIENCE, vol. 92, pp: 410-417, August 2016.

[27] S.V.Manikanthan and D.Sugandhi "Interference Alignment Techniques For Mimo Multicell Based On Relay Interference Broadcast Channel" International Journal of Emerging Technology in Computer Science \& Electronics (IJETCSE) ISSN 0976-1353 Volume- 7, Issue 1 -MARCH 2014. 